\title{
The Spectrum of ROBO3 Mutations in Horizontal Gaze Palsy with Progressive Scoliosis: An Update
}

\author{
Carmine Ungaro ${ }^{1}$, Rosalucia Mazzei ${ }^{2}$, Sebastiano Cavallaro1 ${ }^{1}$, Teresa Sprovieri ${ }^{*}$ \\ ${ }^{1}$ Institute of Neurological Sciences, National Research Council, Mangone, Italy \\ ${ }^{2}$ Institute for Agricultural and Forest Systems in the Mediterranean, National Research Council, Rende, Italy \\ Email: ‘teresa.sprovieri@cnr.it, carmine.ungaro@cnr.it, rosalucia.mazzei@cnr.it, sebastiano.cavallaro@cnr.it
}

How to cite this paper: Ungaro, C., Mazzei, R., Cavallaro, S. and Sprovieri, T. (2018) The Spectrum of ROBO3 Mutations in Horizontal Gaze Palsy with Progressive Scoliosis: An Update. Neuroscience \& Medicine, 9, 187-197.

https://doi.org/10.4236/nm.2018.94019

Received: November 20, 2018

Accepted: December 21, 2018

Published: December 24, 2018

Copyright ( $) 2018$ by authors and Scientific Research Publishing Inc. This work is licensed under the Creative Commons Attribution International License (CC BY 4.0).

http://creativecommons.org/licenses/by/4.0/

\begin{abstract}
Horizontal Gaze Palsy with Progressive Scoliosis is a rare, congenital autosomal recessive disorder caused by mutations in the $R O B O 3$ gene. It is characterized by the absence of conjugate horizontal eye movements with preserved vertical gaze, variable convergence, and progressive scoliosis, developing in childhood and adolescence. $R O B O 3$ gene mutations are causative of the lack, or at least reduction, of crossing of the descending corticospinal and ascending lemniscal sensory tracts in the medulla. To date, 39 different mutations, including missense, nonsense, frameshift, and splice site mutations have been described in the $\mathrm{ROBO}$ gene and related to Horizontal Gaze Palsy With Progressive Scoliosis. In addition, a lot of variants of uncertain pathological significance have been reported for the first time by Illumina Clinical Services. Here we report an update on mutations of the $R O B O 3$ gene and some information on the pathogenesis but much remains to be investigated on the consequences of mutations on $\mathrm{ROBO} 3$ expression and function. Therefore, further detailed functional analyses are necessary to clarify a possible role of the variants of uncertain pathological significance as the cause of the disease. In conclusion, we hope that this article will help in molecular screening for the $R O B O 3$ gene and will contribute to enlargement of the $R O B O 3$ gene variation database.
\end{abstract}

\section{Keywords}

ROBO3, Mutations, HGPPS, Scoliosis

\section{Introduction}

Understanding of the pathogenesis of axonal guidance diseases in recent years 
has improved greatly, and many molecular genetic conditions related to these pathologies have been found. Among them, there is strong interest in Horizontal Gaze Palsy with Progressive Scoliosis (HGPPS; OMIM 607313), firstly described by Dretakis and Kondoyannis in 1974 in consanguineous Greek pedigrees [1], and subsequently reported both in consanguineous pedigrees and unrelated parents of many different ethnicities [2].

\section{Clinical and Genetic Aspects}

HGPPS is a rare, congenital autosomal recessive disorder [3] [4] in which affected individuals are characterized by the absence of conjugate horizontal eye movementswith preserved vertical gaze, variable convergence, and progressive scoliosis. This last disability, starting in infancy or childhood and adolescence, is the most common reason for medical advice in patients with HGPPS and is the most serious condition in terms of function and appearance by which affected patients undergo surgical intervention early in life [5] [6] [7] [8]. No other associated neurological or behavioral abnormalities have been highlighted. To date, 39 different mutations, including missense, nonsense, frameshift, and splice site mutations, in the human $R O B O 3$ gene, have been described and related to HGPPS [5] [9] [10]. This gene is a member of the Roundabout $(R O B O)$ gene family that controls neurite outgrowth, growth cone guidance, and axon fasciculation. $R O B O 3$ gene, encompassing 28 exons and located on chromosomal region 11q23-25, encodes an axon-guidance protein of 1386 amino acid, analogous to mouse Rig1/Rob3, involved in the promoting of midline crossing of neurons in the medulla during brain development [11] [12]. This proteinis predicted to contain an extracellular segment with five immunoglobulin-like domains (Ig1-5) and three fibronectin III-like domains (FnIII1-3), a transmembrane segment $(\mathrm{Tm})$, and an intracellular segment with three cytoplasmic signalling motifs (CC0-3) (Figure 1) [6]. Human $\mathrm{ROBO}$ shares homology with the superfamily of immunoglobulin transmembrane receptors important in axon guidance and neuronal migration, including decussation of developing nerve fiber tracts in the brainstem [5] [13]. Slit proteins 1-3, a family of secreted chemorepellants, are ligands for $R O B O$ proteins and Slit/ROBO interactions regulate myogenesis, leukocyte migration, kidney morphogenesis, angiogenesis, and vasculogenesis in addition to neurogenesis (Figure 2). $R O B O 3$ gene mutations are causative of the lack, or at least reduction, of crossing of the descending corticospinal and ascending lemniscal sensory tracts in the medulla [2] [7] [13]-[20]. Standard MRI T1- and T2-weighted imaging findings consist of pons and cerebellar peduncles hypoplasia, absence of the facial colliculi, butterfly configuration of the medulla and a deep midline pontine cleft [3] [4] [21]. Although scoliosis in HGPPS patients is usually severe, it remains unclear whether the physiopathology of scoliosis is musculoskeletal or neurogenic. Playing $\mathrm{ROBO}$ a critical role for hindbrain axons to appropriately cross the midline [22], a neurogenic mechanism has been postulated by Jen et al. in 2004 [5]. Nevertheless to date, since no 
genotype-phenotype correlation has not been elucidated in HGPPS, possibly because of intra-familial variability of the cardinal features [5] [11] [16], and whereas HGPPS with scoliosis has been described without detectable mutations in $R O B O 3$ gene [14] [23], it is not possible to state that scoliosis is linked to ROBO3 mutations [24].

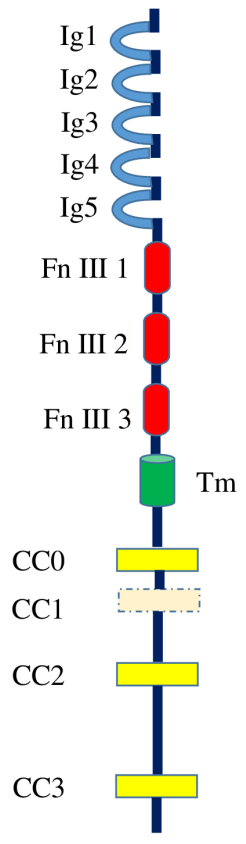

Figure 1. $R O B O 3$ receptor structure. $R O B O 3$ receptor contains an extracellular segment with five immunoglobulin-like domains (Ig1-5) and three fibronectin III-like domains (Fn III 1-3), a transmembrane segment (Tm), and an intracellular segment with three cytoplasmic signalling motifs (CC0-3).

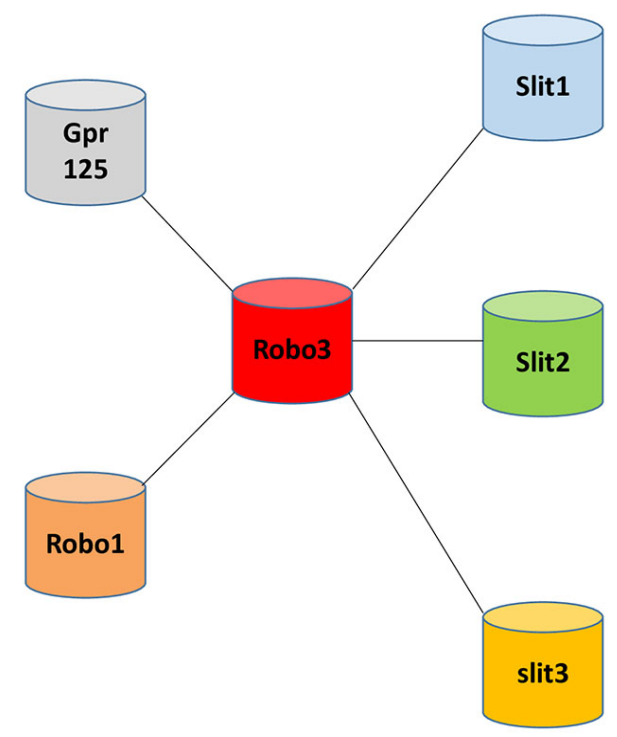

Figure 2. Interacting Proteins for $R O B O 3$ gene. Slit proteins 1-3 and Gpr125 are ligands for $R O B O$ proteins. In particular, Slit proteins 1-3 are a family of secreted chemorepellants and Gpr125 belongs to the family of Adhesion G protein-coupled receptors (GPCRs). 


\section{Spectrum of Mutations in HGPPS}

The purpose of our study is to group all HGPPS-related mutations described to date, both pathogenic and uncertain significance. Mutations are listed in Table 1. HGPPS-related mutations occur in all $R O B O 3$ gene exons and exon-intron boundaries, mostly located on the extracellular part of the protein, inherited both in affected members of consanguineous families harboring homozygous $R O B O 3$ mutations and in individuals from non-consanguineous families harboring compound heterozygous mutations [2]. Most reported $\mathrm{ROBO}$ mutations are scattered throughout the $R O B O 3$ gene without a specific region or domain that can be considered a hot-spot area for mutations [13], although an important accumulation of missense and frameshift mutations in the last exons coding for the C-terminal part of the receptor has been described [25]. This difference, contrasting with HGPPS-associated variants, suggests a critical role of the extracellular domain in $\mathrm{ROBO}$ function. The mutations are highly diverse, and indistinguishable phenotypes result from $R O B O 3$ nonsense, frame-shift, splice-site, or missense mutations, supporting a complete loss of $R O B O 3$ function [26]. Some mutations are predicted to induce a premature stop-codon, associated with expression of a truncated protein or mRNA degradation vianonsense mediated decay (NMD) [27]. In addition, we report in Table 1 a lot of variants of uncertain pathological significance, described for the first time by Illumina Clinical Services (Laboratory Illumina, 2016). It cannot be excluded that they could cause the pathological phenotype, even though experiments are necessary to determine whether these variations underlie the pathogenesis of HGPPS.

Table 1. Currently known mutations in $R O B O 3$ gene linked to HGPPS.

\begin{tabular}{|c|c|c|c|c|c|c|c|}
\hline Variant ID & $\begin{array}{l}\text { Nucleotide } \\
\text { change }\end{array}$ & Exon & $\begin{array}{l}\text { Amino acid } \\
\text { change }\end{array}$ & $\begin{array}{c}\text { Clinical } \\
\text { significance }\end{array}$ & $\begin{array}{c}\text { Control } \\
\text { screened }\end{array}$ & Ethnicity & References \\
\hline rs121918275 & c. $14 \mathrm{~T}>\mathrm{C}$ & 1 & L5P & pathogenic & 106 & Italian & Jen et al., 2004 \\
\hline rs121918276 & c. $196 \mathrm{~A}>\mathrm{C}$ & 2 & I66L & pathogenic & 175 & Greek & Jen et al., 2004 \\
\hline rs121918274 & c. $955 \mathrm{G}>\mathrm{A}$ & 6 & E319K & pathogenic & 197 & Greek & Jen et al., 2004 \\
\hline rs121918271 & $c .2108 G>C$ & 14 & R703P & pathogenic & 150 & Turkish & Jen et al., 2004 \\
\hline rs121918272 & c. $2113 \mathrm{~T}>\mathrm{C}$ & 14 & S705P & pathogenic & 116 & Saudi & Jen et al., 2004 \\
\hline rs121918270 & c. $1082 \mathrm{G}>\mathrm{A}$ & 7 & G361E & pathogenic & 95 & Indian & Jen et al., 2004 \\
\hline rs121918273 & c. $1366 \mathrm{G}>\mathrm{T}$ & 9 & G456X & pathogenic & 95 & Turkish & Jen et al., 2004 \\
\hline CI041652 & c. $2310+1 C$ & 15 & frameshift & pathogenic & 106 & Pakistani & Jen et al., 2004 \\
\hline CI041653 & c. $3325+1 G$ & 23 & frameshift & pathogenic & 116 & Saudi & Jen et al., 2004 \\
\hline CS041545 & c.IVS13 + 1G > A & 13 & frameshift & pathogenic & 93 & Saudi & Jen et al., 2004 \\
\hline rs121918277 & c. $733 \mathrm{C}>\mathrm{T}$ & 4 & $\mathrm{R} 245 \mathrm{~W}$ & pathogenic & 87 & Irish/English & Chan et al., 2006 \\
\hline rs121918278 & c. $2317 \mathrm{C}>\mathrm{T}$ & 15 & Q773X & pathogenic & 87 & Irish/English & Chan et al., 2006 \\
\hline CD061464 & c.1886_1887delTT & 12 & frameshift & pathogenic & 87 & Irish/German & Chan et al., 2006 \\
\hline CD061465 & c.1844_1845delCA & 12 & frameshift & pathogenic & 87 & Irish/German & Chan et al., 2006 \\
\hline
\end{tabular}




\section{Continued}

\begin{tabular}{|c|c|c|c|c|c|c|c|}
\hline CM086900 & c. $2312 \mathrm{C}>\mathrm{T}$ & 15 & P771L & pathogenic & 100 & Saudi & Khan et al., 2008 \\
\hline CM090356 & c. $271 \mathrm{C}>\mathrm{T}$ & 2 & P91S & pathogenic & 50 & Sudanese & $\frac{\text { Abu-Amero et al., }}{\underline{2009}}$ \\
\hline CM090354 & c. $335 \mathrm{G}>\mathrm{C}$ & 2 & $\mathrm{R} 112 \mathrm{P}$ & pathogenic & 120 & Saudi & $\frac{\text { Abu-Amero et al., }}{\underline{2009}}$ \\
\hline rs771613910 & c. $1379 \mathrm{~A}>\mathrm{G}$ & 9 & Q460R & pathogenic & 120 & Saudi & $\begin{array}{l}\text { Abu-Amero et al., } \\
2009\end{array}$ \\
\hline HM070122 & c. $1726 \mathrm{~T}>\mathrm{C}$ & 11 & W576R & pathogenic & 120 & Saudi & $\frac{\text { Abu-Amero et al., }}{\underline{2009}}$ \\
\hline CD090357 & c.571delC & 2 & frameshift & pathogenic & 120 & Saudi & $\begin{array}{l}\text { Abu-Amero et al., } \\
2009\end{array}$ \\
\hline CM095023 & c. $1450 \mathrm{~T}>\mathrm{C}$ & 9 & W484R & pathogenic & NR & Tunisian & Amouri et al., 2009 \\
\hline CM095022 & c. $283 \mathrm{~T}>\mathrm{C}$ & 2 & I95T & pathogenic & 100 & Tunisian & Amouri et al., 2009 \\
\hline CD095024 & c.1618delG & 10 & R539fsX574 & pathogenic & NR & Tunisian & Amouri et al., 2009 \\
\hline rs121918277 & c. $733 \mathrm{C}>\mathrm{T}$ & 4 & $\mathrm{R} 245 \mathrm{~W}$ & pathogenic & NR & Tunisian & Amouri et al., 2009 \\
\hline CD118835 & $\begin{array}{c}\text { c. } 2 \_16 \\
\text { delTGCTGCGCTACCTGC }\end{array}$ & 1 & & pathogenic & 100 & Saudi & $\begin{array}{l}\text { Abu-Amero et al., } \\
2011\end{array}$ \\
\hline CX115641 & c.913delAinsTGC & 6 & I305CfsX13 & pathogenic & 144 & Caucasian/Turkish & Volk et al., 2011 \\
\hline CS115643 & c. $3319 \mathrm{~A}>\mathrm{C}$ & 22 & $\begin{array}{c}\text { skip }+ \\
\text { frameshift }\end{array}$ & pathogenic & 144 & Caucasian/Turkish & Volk et al., 2011 \\
\hline CG115642 & $\begin{array}{l}\text { c.2769_2779del11, } \\
2779+1 \_+20 \operatorname{del} 20\end{array}$ & 17 & & pathogenic & 144 & Caucasian/Turkish & Volk et al., 2011 \\
\hline NA & $\mathrm{G}>\mathrm{T}$ & 17 & $\bullet \mathrm{E}-\mathrm{X}$ & pathogenic & NR & Indian & Ng et al., 2011 \\
\hline rs34519996 & c.541dup & 3 & E181GfsX71 & pathogenic & NR & Kosovar & Kurian et al., 2013 \\
\hline NA & c. $2663 \mathrm{~T}>\mathrm{C}$ & 17 & L888P & pathogenic & NR & Saudi & $\begin{array}{c}\text { Khan and } \\
\text { Abu-Amero, } 2014\end{array}$ \\
\hline NA & c.767_776delAGCGTCCCTC & 5 & & pathogenic & NR & Portuguese & Pina et al. 2014 \\
\hline NA & c.767-2_767-1delAG & 5 & & pathogenic & NR & Portuguese & Pina et al. 2014 \\
\hline NA & $c .2392 \mathrm{C}>\mathrm{T}$ & 15 & Q798X & pathogenic & NR & Japanese & Yamada et al., 2015 \\
\hline NA & c.2576del & 16 & P859fs & pathogenic & NR & Austrian & Arlt et al., 2015 \\
\hline NA & c. $1158 \mathrm{G}>\mathrm{C}$ & 7 & Q386H & pathogenic & NR & Spanish & $\begin{array}{l}\text { Fernández-Vega } \\
\text { Cueto et al., } 2016\end{array}$ \\
\hline NA & $c .416 G>T$ & 2 & G139V & pathogenic & NR & Switzerland & $\begin{array}{l}\text { Hackenberg et al., } \\
2017\end{array}$ \\
\hline NA & $c .2524 \mathrm{C}>\mathrm{T}$ & 16 & $\mathrm{R} 842 \mathrm{X}$ & pathogenic & NR & Turkish & $\begin{array}{l}\text { Bozdoğan et al., } \\
\qquad 2017\end{array}$ \\
\hline NA & IV55-5delCATAG & 2 & & pathogenic & NR & Cape Verde & $\begin{array}{c}\text { Mendes Marques et } \\
\text { al., } 2017\end{array}$ \\
\hline NA & c.767_775delAGCGTCCCT & 5 & & pathogenic & NR & Cape Verde & $\begin{array}{c}\text { Mendes Marques et } \\
\text { al., } 2017\end{array}$ \\
\hline NA & c. $1433 \mathrm{C}>\mathrm{T}$ & 9 & P478L & pathogenic & 100 & Italian & Ungaro et al., 2018 \\
\hline NA & c. $3321-\mathrm{G}>\mathrm{A}$ & int 22 & Splice site mut & pathogenic & 100 & Italian & Ungaro et al., 2018 \\
\hline
\end{tabular}




\section{Continued}

\begin{tabular}{|c|c|c|c|c|c|c|c|}
\hline rs 185584218 & c. $-120 \mathrm{G}>\mathrm{C}$ & & \multicolumn{2}{|c|}{ 5' UTR variant uncertain significance } & NR & NR & $\begin{array}{l}\text { Illumina Clinical } \\
\text { Services Laboratory, } \\
\text { Illumina, } 2016\end{array}$ \\
\hline rs886047914 & c. ${ }^{\star} 68 \mathrm{C}>\mathrm{G}$ & & \multicolumn{2}{|c|}{ 3'UTR variant uncertain significance } & NR & NR & $\begin{array}{l}\text { Illumina Clinical } \\
\text { Services Laboratory, } \\
\text { Illumina, } 2016\end{array}$ \\
\hline rs114572753 & c. $1189 \mathrm{C}>\mathrm{A}$ & 8 & P397T & uncertain significance & NR & NR & $\begin{array}{l}\text { Illumina Clinical } \\
\text { Services Laboratory, } \\
\text { Illumina, } 2016\end{array}$ \\
\hline rs114572753 & c. $1189 \mathrm{C}>\mathrm{T}$ & 8 & P397S & uncertain significance & NR & NR & $\begin{array}{l}\text { Illumina Clinical } \\
\text { Services Laboratory, } \\
\text { Illumina, } 2016\end{array}$ \\
\hline rs543770866 & c. $-60 \mathrm{G}>\mathrm{T}$ & & \multicolumn{2}{|c|}{ 5' UTR variant uncertain significance } & NR & NR & $\begin{array}{l}\text { Illumina Clinical } \\
\text { Services Laboratory, } \\
\text { Illumina, } 2016\end{array}$ \\
\hline rs774646580 & c. $43 \mathrm{~T}>\mathrm{C}$ & 1 & F15L & uncertain significance & NR & NR & $\begin{array}{l}\text { Illumina Clinical } \\
\text { Services Laboratory, } \\
\text { Illumina, } 2016\end{array}$ \\
\hline rs189616702 & c. $46 \mathrm{G}>\mathrm{A}$ & 1 & A16T & uncertain-significance & NR & NR & $\begin{array}{l}\text { Illumina Clinical } \\
\text { Services Laboratory, } \\
\text { Illumina, } 2016\end{array}$ \\
\hline rs145440217 & c. $160+12 \mathrm{C}>\mathrm{T}$ & int & & uncertain significance & NR & NR & $\begin{array}{l}\text { Illumina Clinical } \\
\text { Services Laboratory, } \\
\text { Illumina, } 2016\end{array}$ \\
\hline NA & c. $.568 \mathrm{C}>\mathrm{T}$ & & P190S & uncertain significance & NR & NR & $\begin{array}{l}\text { Illumina Clinical } \\
\text { Services Laboratory, } \\
\text { Illumina, } 2016\end{array}$ \\
\hline rs192622083 & c. $592 \mathrm{G}>\mathrm{A}$ & 3 & V198M & uncertain significance & NR & NR & $\begin{array}{l}\text { Illumina Clinical } \\
\text { Services Laboratory, } \\
\text { Illumina, } 2016\end{array}$ \\
\hline rs765531515 & c. $716 \mathrm{C}>\mathrm{T}$ & 4 & S239F & uncertain significance & NR & NR & $\begin{array}{l}\text { Illumina Clinical } \\
\text { Services Laboratory, } \\
\text { Illumina, } 2016\end{array}$ \\
\hline rs747047729 & c. $764 \mathrm{~T}>\mathrm{C}$ & 4 & L255P & uncertain significance & NR & NR & $\begin{array}{l}\text { Illumina Clinical } \\
\text { Services Laboratory, } \\
\text { Illumina, } 2016\end{array}$ \\
\hline rs200451819 & c. $769 \mathrm{C}>\mathrm{T}$ & 15 & $\mathrm{R} 257 \mathrm{C}$ & uncertain significance & NR & NR & $\begin{array}{l}\text { Illumina Clinical } \\
\text { Services Laboratory, } \\
\text { Illumina, } 2016\end{array}$ \\
\hline rs142090631 & c. $850 \mathrm{G}>\mathrm{A}$ & 5 & $\mathrm{D} 284 \mathrm{~N}$ & uncertain significance & NR & NR & $\begin{array}{l}\text { Illumina Clinical } \\
\text { Services Laboratory, } \\
\text { Illumina, } 2016\end{array}$ \\
\hline rs151168595 & c. $968 \mathrm{C}>\mathrm{T}$ & 20 & $\mathrm{~T} 323 \mathrm{M}$ & uncertain significance & NR & NR & $\begin{array}{l}\text { Illumina Clinical } \\
\text { Services Laboratory, } \\
\text { Illumina, } 2016\end{array}$ \\
\hline rs886047908 & c. $1034-13 \mathrm{C}>\mathrm{G}$ & 7 & & uncertain significance & NR & NR & $\begin{array}{l}\text { Illumina Clinical } \\
\text { Services Laboratory, } \\
\text { Illumina, } 2016\end{array}$ \\
\hline rs200197609 & c. $1542 \mathrm{G}>\mathrm{A}$ & 10 & M514I & uncertain significance & NR & NR & $\begin{array}{l}\text { Illumina Clinical } \\
\text { Services Laboratory, } \\
\text { Illumina, } 2016\end{array}$ \\
\hline
\end{tabular}




\section{Continued}

\begin{tabular}{|c|c|c|c|c|c|c|c|}
\hline rs550454340 & c. $1957 \mathrm{~A}>\mathrm{C}$ & 13 & S653R & uncertain significance & NR & NR & $\begin{array}{l}\text { Illumina Clinical } \\
\text { Services Laboratory, } \\
\text { Illumina, } 2016\end{array}$ \\
\hline rs778522624 & c. $2048 \mathrm{C}>\mathrm{T}$ & 13 & P683L & uncertain significance & NR & NR & $\begin{array}{l}\text { Illumina Clinical } \\
\text { Services Laboratory, } \\
\text { Illumina, } 2016\end{array}$ \\
\hline rs184921255 & c. $2102 \mathrm{G}>\mathrm{T}$ & 14 & G701V & uncertain significance & NR & NR & $\begin{array}{l}\text { Illumina Clinical } \\
\text { Services Laboratory, } \\
\text { Illumina, } 2016\end{array}$ \\
\hline rs189303564 & c. $2183 \mathrm{G}>\mathrm{A}$ & 14 & S728N & uncertain significance & NR & NR & $\begin{array}{l}\text { Illumina Clinical } \\
\text { Services Laboratory, } \\
\text { Illumina, } 2016\end{array}$ \\
\hline rs372821877 & c. $2427 \mathrm{G}>\mathrm{A}$ & 16 & W809X & pathogenic & NR & NR & $\begin{array}{c}\text { EGL Genetic } \\
\text { Diagnostics, } \\
\text { Eurofins Clinical } \\
\text { Diagnostics, } 2017\end{array}$ \\
\hline rs536588537 & c. $2504 \mathrm{~T}>\mathrm{C}$ & 16 & L835P & uncertain significance & NR & NR & $\begin{array}{l}\text { Illumina Clinical } \\
\text { Services Laboratory, } \\
\text { Illumina, } 2016\end{array}$ \\
\hline rs199932669 & c. $2621 \mathrm{~T}>\mathrm{A}$ & 17 & L874Q & uncertain significance & NR & NR & $\begin{array}{l}\text { Illumina Clinical } \\
\text { Services Laboratory, } \\
\text { Illumina, } 2016\end{array}$ \\
\hline rs756785207 & c. $2779+9 \mathrm{C}>\mathrm{T}$ & int & & uncertain significance & NR & NR & $\begin{array}{l}\text { Illumina Clinical } \\
\text { Services Laboratory, } \\
\text { Illumina, } 2016\end{array}$ \\
\hline$\underline{\text { rs763859563 }}$ & c. $2931 \mathrm{~A}>\mathrm{T}$ & 20 & E977D & uncertain significance & NR & NR & $\begin{array}{l}\text { Illumina Clinical } \\
\text { Services Laboratory, } \\
\text { Illumina, } 2016\end{array}$ \\
\hline rs765206958 & c. $2947 \mathrm{~T}>\mathrm{C}$ & 20 & C983R & uncertain significance & NR & NR & $\begin{array}{l}\text { Illumina Clinical } \\
\text { Services Laboratory, } \\
\text { Illumina, } 2016\end{array}$ \\
\hline rs75098003 & c. $2993 \mathrm{G}>\mathrm{T}$ & 21 & G998V & uncertain significance & NR & NR & $\begin{array}{l}\text { Illumina Clinical } \\
\text { Services Laboratory, } \\
\text { Illumina, } 2016\end{array}$ \\
\hline rs886047909 & c. $3091 \mathrm{~A}>\mathrm{C}$ & 21 & T1031P & uncertain significance & NR & NR & $\begin{array}{l}\text { Illumina Clinical } \\
\text { Services Laboratory, } \\
\text { Illumina, } 2016\end{array}$ \\
\hline rs886047910 & c. $3139 \mathrm{~T}>\mathrm{G}$ & 21 & W1047G & uncertain significance & NR & NR & $\begin{array}{l}\text { Illumina Clinical } \\
\text { Services Laboratory, } \\
\text { Illumina, } 2016\end{array}$ \\
\hline rs761311616 & c. $3320+12 \mathrm{C}>\mathrm{T}$ & int & & uncertain significance & NR & NR & $\begin{array}{l}\text { Illumina Clinical } \\
\text { Services Laboratory, } \\
\text { Illumina, } 2016\end{array}$ \\
\hline rs886047912 & c. $3706 \mathrm{G}>\mathrm{A}$ & 25 & G1236R & uncertain significance & NR & NR & $\begin{array}{l}\text { Illumina Clinical } \\
\text { Services Laboratory, } \\
\text { Illumina, } 2016\end{array}$ \\
\hline rs886047913 & c. $3707 \mathrm{G}>\mathrm{T}$ & 25 & G1236V & uncertain significance & NR & NR & $\begin{array}{l}\text { Illumina Clinical } \\
\text { Services Laboratory, } \\
\text { Illumina, } 2016\end{array}$ \\
\hline
\end{tabular}




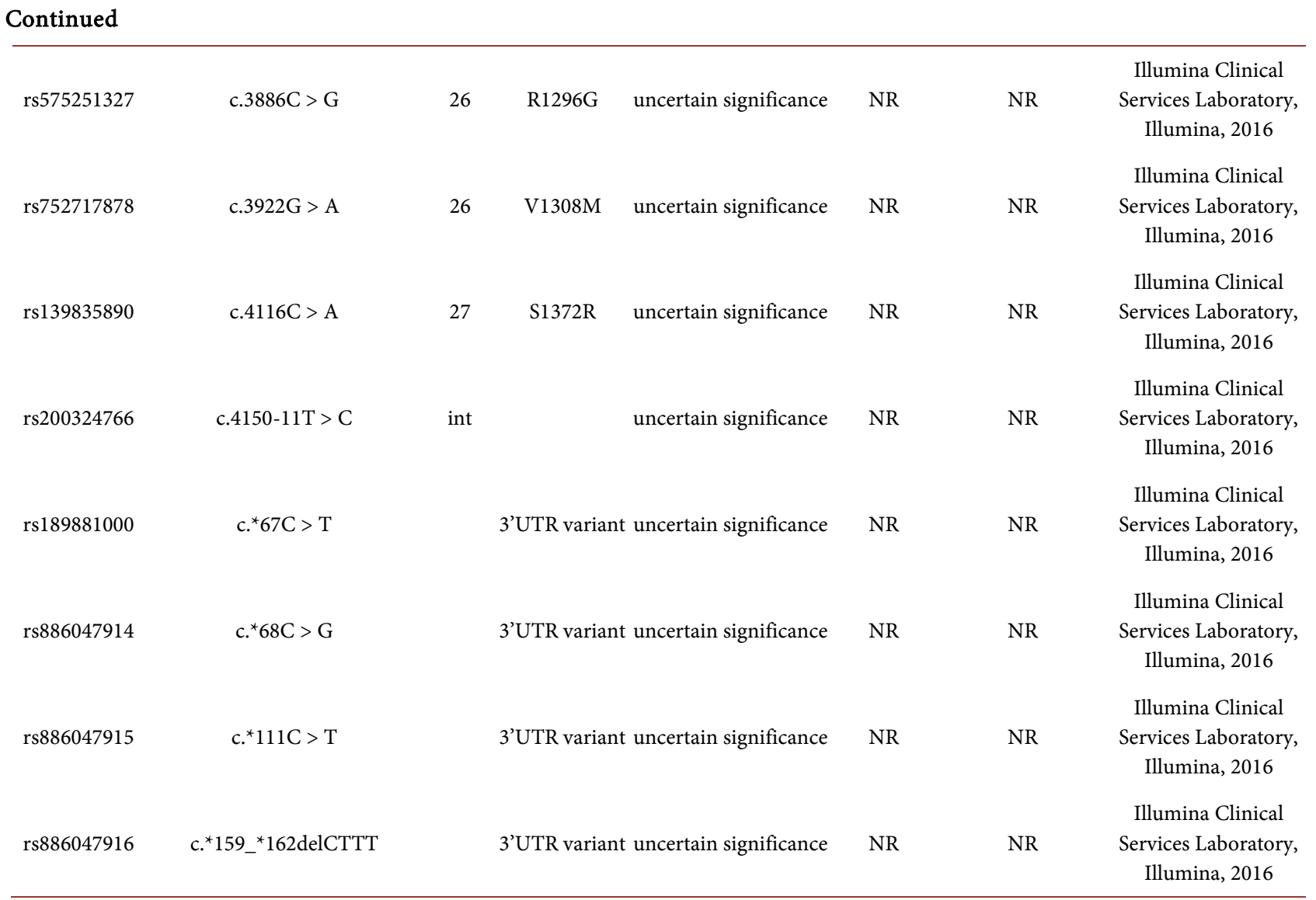

- The nucleotide number where the mutation was located was not mentioned by the authors. $\bullet$ The codon number was not mentioned by the authors. Mutations in bold were inherited in heterozygous status; mutations in Italics and bold were inherited both in heterozygous status as compound and in heterozygous status. The remaining mutations were inherited in homozygous status. NA $=$ Not available. NR $=$ Not reported by authors.

\section{Discussion and Conclusion}

$R O B O 3$ is a transmembrane receptor that plays an important role in axon guidance and neuronal migration and is critical for long ascending medial lemniscal and descending corticospinal tracts in the medulla. In fact, in HGPPS contralateral axon projections of inter-nuclear neurons that co-ordinate the activity of oculomotor and abducens neurons fail to form, resulting in defects in horizontal eye movements. Mutations in $R O B O 3$ are associated with noncrossing of selected paths in the central nervous system that are normally subjected to midline crossing during embryonic development [20]. A clinical misdiagnosis of HGPPS seems unlikely because the association of horizontal gaze palsy and severe scoliosis is considered pathognomonic of HGPPS [5] [11]. Diffusion tensor imaging (DTI) and DTI tractography have to be performed to evaluate the corticospinal pathways and to confirm the presence of uncrossed corticospinal tracts, being a useful adjunct to the structural magnetic resonance imaging (MRI) in confirming the clinical features suggestive of HGPPS. Since an early diagnosis of HGPPS is possible by analysis of the $R O B O 3$ gene, this is of great importance with respect to genetic counselling of HGPPS families [19]. A genotypic assessment of 
$R O B O 3$ mutations presents challenges because relatively little is known about the function of various $R O B O 3$ domains or actions of alternative splice forms of $R O B O 3$ in the human Brainstem [5] [13] [28]. The action of $R O B O 3$ or its protein product might be inhibited by environmental or epigenetic factors in the developing brainstem; furthermore, a phenotype identical to HGPPS might be caused by abnormalities in $R O B O 3$ splice variant expression. Moreover,although most reported $\mathrm{ROBO} 3$ mutations are equally distributed along the $\mathrm{ROBO}$ sequence, it would be interesting to determine whether specific mutation types are associated with a more disease phenotype and/or whether other disease genes for patients with horizontal gaze palsy with or without scoliosis who do not harbor mutations in $\mathrm{ROBO} 3$ are engaged. Consequentially, a Targeted Next Generation Sequencing (NGS) could be useful to elucidate the possible contribute of other genes. In fact, despite the advances in understanding about this condition much remains to be investigated on the consequences of mutations on $R O B O 3$ expression and function. Further detailed functional analyses are necessary to clarify a possible role of the variants of uncertain pathological significance as causative of the disease. In conclusion, we hope that this article will help in molecular screening for the $\mathrm{ROBO}$ gene and will contribute to enlargement of the $\mathrm{ROBO} 3$ gene variation database.

\section{Funding}

No funding was received for this work.

\section{Conflicts of Interest}

The authors declare no conflicts of interest regarding the publication of this paper.

\section{References}

[1] Dretakis, E.K. and Kondoyannis, P.N. (1974) Congenital Scoliosis Associated with Encephalopathy in Five Children of Two Families. The Journal of Bone \& Joint Surgery, 56, 1747-1750. https://doi.org/10.2106/00004623-197456080-00034

[2] Chan, W.M., Traboulsi, E.I., Arthur, B., et al. (2006) Horizontal Gaze Palsy with Progressive Scoliosis Can Result from Compound Heterozygous Mutations in ROBO3. Journal of Medical Genetics, 43, e11. https://doi.org/10.1136/jmg.2005.035436

[3] Rossi, A., Catala, M., Biancheri, R., et al. (2004) MR Imaging of Brain-Stem Hypoplasia in Horizontal Gaze Palsy with Progressive Scoliosis. American Journal of Neuroradiology, 25, 1046-1048.

[4] dos Santos, A.V., Matias, S., Saraiva, P. and Goulão, A. (2006) MR Imaging Features of Brain Stem Hypoplasia in Familial Horizontal Gaze Palsy and Scoliosis. American Journal of Neuroradiology, 27, 1382-1383.

[5] Jen, J.C., Chan, W.M., Bosley, T.M., et al. (2004) Mutations in a Human ROBO Gene Disrupt Hindbrain Axon Pathway Crossing and Morphogenesis. Science, 304, 1509-1513.

[6] Jen, J.C. (2008) Effects of Failure of Development of Crossing Brainstem Pathways 
on Ocular Motor Control. Progress in Brain Research, 171, 137-141. https://doi.org/10.1016/S0079-6123(08)00618-3

[7] Ng, A.S., Sitoh, Y.Y., Zhao, Y., et al. (2011) Ipsilateral Stroke in a Patient with Horizontal Gaze Palsy with Progressive Scoliosis and a Subcortical Infarct. Stroke, 42, e1-e3.

[8] Irahara, K., Saito, Y., Sugai, K., et al. (2014) Pontine Malformation, Undecussated Pyramidal Tracts, and Regional Polymicrogyria: A New Syndrome. Pediatric neurology, 50, 384-288. https://doi.org/10.1016/j.pediatrneurol.2013.12.013

[9] Jen, J., Coulin, C.J., Bosley, T.M., et al. (2002) Familial Horizontal Gaze Palsy with Progressive Scoliosis Maps to Chromosome 11q23-25. Neurology, 59, 432-435. https://doi.org/10.1212/WNL.59.3.432

[10] Bozdoğan, S.T., Dinç, E., Sari, A.A., et al. (2017) A Novel Mutation of ROBO3 in Horizontal Gaze Palsy with Progressive Scoliosis. Ophthalmic Genetics, 38, 284-285. https://doi.org/10.1080/13816810.2016.1188123

[11] Bosley, T.M., Salih, M.A., Jen, J.C., et al. (2005) Neurologic Features of Horizontal Gaze Palsy and Progressive Scoliosis with Mutations in ROBO3. Neurology, 64, 1196-1203. https://doi.org/10.1212/01.WNL.0000156349.01765.2B

[12] Sabatier, C., Plump, A.S., Ma, L., et al. (2004) The Divergent Robo Family Protein Rig-1/Robo3 Is a Negative Regulator of Slit Responsiveness Required for Midline Crossing by Commissural axons. Cell, 117, 157-169. https://doi.org/10.1016/S0092-8674(04)00303-4

[13] Abu-Amero, K.K., Kapoor, S., Hellani, A., et al. (2011) Horizontal Gaze Palsy and Progressive Scoliosis Due to a Deleterious Mutation in ROBO3. Ophthalmic Genetics, 32, 231-236. https://doi.org/10.3109/13816810.2011.580445

[14] Abu-Amero, K.K., Faletra, F., Gasparini, P., et al. (2011) Horizontal Gaze Palsy and Progressive Scoliosis without ROBO3 Mutations. Ophthalmic Genetics, 32, 212-216. https://doi.org/10.3109/13816810.2011.574186

[15] Yamada, S., Okita, Y., Shofuda, T., et al. (2015) Ipsilateral Hemiparesis Caused by Putaminal Hemorrhage in a Patient with Horizontal Gaze Palsy with Progressive Scoliosis: A Case Report. BMC Neurology, 15, 25.

[16] Abu-Amero, K.K., al Dhalaan, H., al Zayed, Z., et al. (2009) Five New Consanguineous Families with Horizontal Gaze Palsy and Progressive Scoliosis and Novel ROBO3 Mutations. Journal of the Neurological Sciences, 276, 22-26.

[17] Amouri, R., Nehdi, H., Bouhlal, Y., et al. (2009) Allelic ROBO3 Heterogeneity in Tunisian Patients with Horizontal Gaze Palsy with Progressive Scoliosis. Journal of Molecular Neuroscience, 39, 337-341. https://doi.org/10.1007/s12031-009-9217-4

[18] Khan, A.O., Oystreck, D.T., Al-Tassan, N., et al. (2008) Bilateral Synergistic Convergence Associated with Homozygous ROB03 Mutation (p.Pro771Leu). Ophthalmology, 115, 2262-2265. https://doi.org/10.1016/j.ophtha.2008.08.010

[19] Kurian, M., Megevand, C., De Haller, R., et al. (2013) Early-Onset or Rapidly Progressive Scoliosis in Children: Check the Eyes! European Journal of Paediatric Neurology, 17, 671-675. https://doi.org/10.1016/j.ejpn.2013.05.011

[20] Volk, A.E., Carter, O., Fricke, J., et al. (2011) Horizontal Gaze Palsy with Progressive Scoliosis: Three Novel ROBO3 Mutations and Descriptions of the Phenotypes of Four Patients. Molecular Vision, 17, 1978-1986.

[21] Bomfim, R.C., Távora, D.G., Nakayama, M. and Gama, R.L. (2009) Horizontal Gaze Palsy with Progressive Scoliosis: CT and MR Findings. Pediatric Radiology, 39,184-187. https://doi.org/10.1007/s00247-008-1058-8 
[22] Graeber, C.P., Hunter, D.G. and Engle, E.C. (2013) The Genetic Basis of Incomitant Strabismus: Consolidation of the Current Knowledge of the Genetic Foundations of Disease. Seminars in Ophthalmology, 28, 427-437. https://doi.org/10.3109/08820538.2013.825288

[23] Ungaro, C., Valentini, S., Cerasa, A., et al. (2018) Horizontal Gaze Palsy with Progressive Scoliosis: Two Novel ROBO3 Mutations in a Compound Heterozygous Sporadic Case. Journal of Neuro-Ophthalmology, 38, 131-132.

[24] Ungaro, C., Mazzei, R. and Sprovieri, T. (2018) Horizontal Gaze Palsy with Progressive Scoliosis: Is Scoliosis Linked to ROBO3 Mutations? Neurological Sciences, $1-2$.

[25] Auton, A., Brooks, L.D., et al. (2015) A Global Reference for Human Genetic Variation. Nature, 526, 68-74.

[26] Engle, E.C. (2010) Human Genetic Disorders of Axon Guidance. Cold Spring Harbor Perspectives in Biology, 2, a001784. https://doi.org/10.1101/cshperspect.a001784

[27] Friocourt, F. and Chédotal, A. (2017) The Robo3 Receptor, a Key Player in the Development, Evolution, and Function of Commissural Systems. Developmental Neurobiology, 77, 876-890. https://doi.org/10.1002/dneu.22478

[28] Evans, T.A. and Bashaw, G.J. (2010) Functional Diversity of Robo Receptor Immunoglobulin Domains Promotes Distinct Axon Guidance Decisions. Current Biology, 20, 567-572. https://doi.org/10.1016/j.cub.2010.02.021 